\title{
Validity of Darcy's Law Under Transient Conditions
}

U.S. GEOLOGICAL SURVEY PROFESSIONAL PAPER 1331

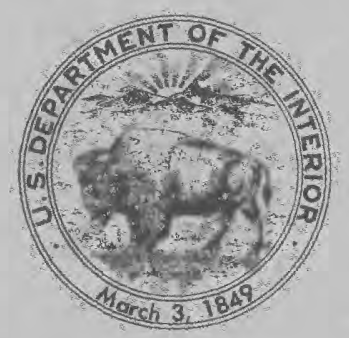




\section{Validity of Darcy's Law Under Transient Conditions}

$B y$ CHARLES E. MONGAN

U.S. GEOLOGICAL SURVEY PROFESSIONAL PAPER 1331

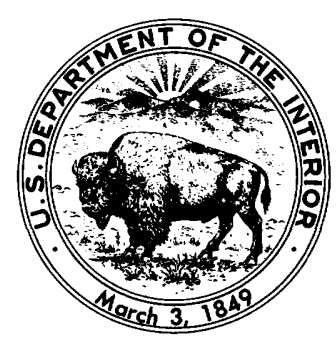




\section{DEPARTMENT OF THE INTERIOR}

WILLIAM P. CLARK, Secretary

\section{U.S. GEOLOGICAL SURVEY}

Dallas L. Peck, Director

For sale by the Distribution Branch, U.S. Geological Survey, 604 South Pickett Street, Alexandria, VA 22304 


\section{CONTENTS}

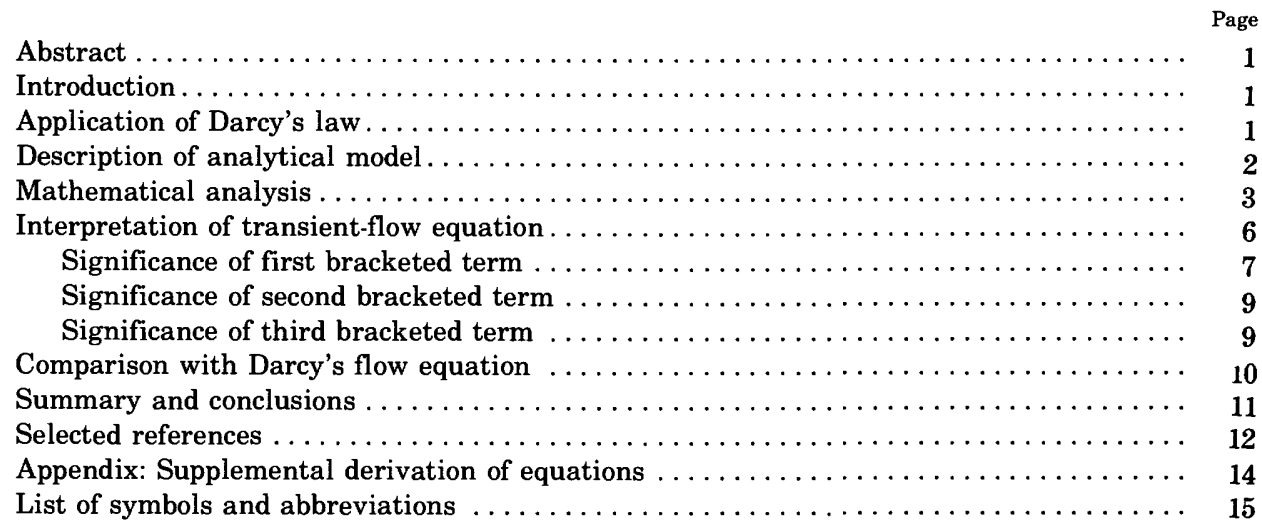

\section{ILLUSTRATIONS}

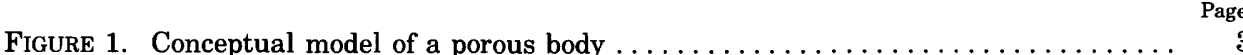

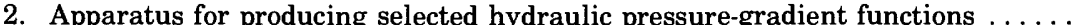

3. Relation between time constant and radius of flow tube $\ldots \ldots \ldots \ldots \ldots \ldots, 8$

4. Comparison between Darcy and transient-flow equations for indicated step increase

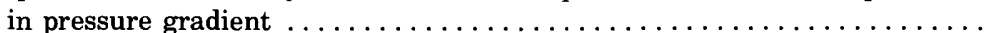

TABLE

TABLE 1. Short table of finite Hankel transforms 



\title{
VALIDITY OF DARCY'S LAW UNDER TRANSIENT CONDITIONS
}

\author{
By Charles E. MONGAN
}

\begin{abstract}
Darcy's law, which describes fluid flow through porous materials, was developed for steady-flow conditions. The validity of applying this law to transient flows has been mathematically verified for most ground-water flow conditions. The verification was accomplished through application of Hankel transforms to linearized Navier-Stokes equations which described flow in a small-diameter cylindrical tube chosen to represent a single pore in a porous medum.
\end{abstract}

\section{INTRODUCTION}

When fluid flows are studied, the assumption is frequently made that the flow is in the steady state. However, most flows in nature are in transient states. Flows in aquifers and oil-bearing strata are examples of such transient flows occurring in porous media. Flows in porous media are governed by Darcy's law under steady-state conditions. This same law is assumed to be valid with transient flows. The purpose of this report is to develop a transient-flow equation from fundamental physical laws and to compare the results from such an equation with the results obtained from an empirical Darcy's law. An equality of results will verify that Darcy's law may be applied with assurance to most transient-flow conditions.

To treat the problem mathematically, certain idealizations are necessary. These will be discussed in detail in the following sections.

\section{APPLICATION OF DARCY'S LAW}

Darcy's law (1856) states that the volumetric discharge of fluid through a porous body is proportional to the saturated cross-sectional area perpendicular to the direction of flow and is inversely proportional to the length of the flow path. It also is proportional to the difference in hydraulic head between the input and output surfaces.

Darcy's law may be written (Hubbert, 1969, p. 31):

$$
Q=-K A \frac{h_{2}-h_{1}}{L},
$$

where

Description Dimensions

$Q=$ volumetric flow rate $\quad L^{3} T^{-1}$

$A=$ cross-sectional area of porous medium perpendicular to direction of flow $\quad L^{2}$

$K=$ hydraulic conductivity of porous medium

$h=$ hydraulic head

$L=$ length of flow path

Darcy's formulation (eq. 1) followed from his numerous experiments. Many subsequent experiments, both in the laboratory and in the field, have shown its validity for steady-state conditions. Because of the law's importance, both for the management of ground-water resources and for its relevance to petroleum production, vigorous efforts have been made to give it a substantial theoretical basis (Gray and O'Neill, 1976).

Following these efforts, it is now customary to write the equation in a slightly different form; Hubbert (1969, p. 59) writes:

$$
q=-\sigma \frac{d \phi}{d L}
$$

where, in addition to terms previously defined,

Description

$q=$ specific rate of fluid flow

$\sigma=$ specific fluid conductivity

$\phi=$ fluid potential; $\phi=g h$

$g=$ acceleration (rate and direc-

tion) due to gravity

Scheidegger (1964) writes as follows:

$$
q=-\frac{k}{\mu}[\operatorname{grad} p-\varrho g],
$$

Dimensions

$L T^{-1}$

$L T^{-1}$

$L^{2} T^{-2}$

$L T^{\mathbf{2}}$ 
where, in addition to the terms previously defined,

\section{Description}

$\begin{array}{ll}\mu=\text { dynamic viscosity of fluid } & M L^{-1} T^{-1} \\ \varrho=\text { density of fluid } & M L^{-3} \\ k=\text { intrinsic permeability of } \\ \quad \text { porous medium (see Lohman, } \\ \begin{array}{l}\text { 1972) } \\ p=\text { hydraulic pressure at a given } \\ \quad \text { point }\end{array} & L^{2} \\ & M L^{-1} T^{-2}\end{array}$

As Hubbert points out, the form of Darcy's law is similar to that of the other transport laws-namely, Ohm's law, Fourier's law, and Ficks' law, which pertain, respectively, to the flow of electrical current, the flow of heat, and the diffusion of chemical concentration. In all these cases, the laws apply to the steady state.

In the steady state, a flow field is characterized by the constancy of the velocity components at each given point in the field. In the unsteady state, the velocities at a given point change with time. A change with time implies a change of momentum, which, of course, involves the density of the fluid.

The absence in the Darcy equation of any explicit variable for time and the absence of density in a dynamical context indicate that the Darcy equation is an approximation for the unsteady state. Although it has been clear for some time that the law is an approximation, the real question continues to be: "How good an approximation is it?" The intent in this paper is to set the stage for further numerical estimates of the error made in using Darcy's law in its customary form with respect to current field applications.

The present problem is to obtain a better estimate of the error resulting from neglecting the inertial aspects of the transient-flow process. This paper seeks to furnish such an estimate on the basis of an analytical study of a simple flow-field element. From such an element, an approximation to a porous body may be constructed, at least conceptually.

A customary approach to a mathematical analysis such as that discussed in this report is to define a geometric model of the physical circumstances that are to be studied. Of course, it is necessary to examine the model in some detail to be sure that it properly reflects reality. The model should be simple enough so that it is amenable to direct mathematical description and calculation.

\section{DESCRIPTION OF ANALYTICAL MODEL}

There are certain properties that characterize porous bodies, one of which is the presence of continuous passages through the material of the body that are capable of conducting fluid. The passages do not have constant cross-sectional areas-they change both in area and direction. In addition, they may be characterized by a large surface area-to-volume ratio. The surface is the areal extent of the rock passage that is actually wetted by the liquid, and the volume is the volume of liquid contained within that surface.

Over the years, various attempts to design mathematical models capable of describing in detail the flow of fluid through porous earth materials have been made. Brief mention will be made of some of these to give the tenor of the analytical developments up to the present.

Happel and Brenner (1965, p. 389) make reference to an earlier survey of the literature on the efforts to describe Darcy's law from fundamental principles. Their review can be highlighted by the following three summary paragraphs:

1. Slichter (1899) used an arrangement of spheres to idealize a porous earth material and assumed a triangular cross section for a flow passage. It was assumed that Poiseuille's law could be applied to describe fluid flow in the triangular section. However, this model was inadequate for general use because it was oversimplified.

2. Blake (1922) introduced the concept of a hydraulic radius, which he defined as the volume filled with the liquid divided by the wetted surface. He regarded a flow passage in a porous body as a tube having a very complicated cross section. Using the hydraulic radius so defined, Blake was able to achieve results that were quite useful. It should be noted that Blake's hydraulic radius is the inverse of the surface area-to-volume ratio that plays a considerable role in catalytic chemistry-particularly in chemical engineering.

3. Additional mathematical models were designed by Kozeny (1953), and a semiempirical model was made by Carman in 1956. The Kozeny-Carman work led to expressions for the constant in the Darcy equation which could be related to the void space and the hydraulic radius. Empirical developments of these constants are now commonly used in chemical engineering to analyze flow problems.

Happel and Brenner themselves made an analysis based on similitude and came up with constants for the flow equation that showed that the Darcy constant is dimensionally proportional to $L$ squared divided by a constant $C$. This was a useful advance.

In a later study, Scheidegger (1964) made an analysis based on probability considerations. The pore structure 
was supposed to have a random distribution which could be given in probability terms. The motion of a fluid particle through such a flow field was assumed to be a random walk. Scheidegger made some use of the Poiseuille concepts.

Scheidegger makes the comments that the earlier lines of analytical development were twofold: (1) mathematical models based on probability, and (2) models based on the geometrical stacking of flowpassage components.

Many ground-water investigators since 1965 have examined a wide variety of flow problems and have published the results of their theoretical derivations. Because none of the foregoing investigative studies permit the Darcy constant to be calculated for an actual assemblage of porous earth particles, the fluid-flow analysis continues to rest on a somewhat empirical basis.

This paper combines some of the respective probabilistic and geometric analytical features. As can be seen in figure 1 , it is possible to simulate a porous body by assembling a bundle of capillary tubes. If the crosssectional area of each tube is described by a Gaussian distribution, then we have achieved a random collection of the areas through which the liquid moves. A representative sample of such a collection can then be made in the three-dimensional form of a small elemental "pancake" (fig. 1).

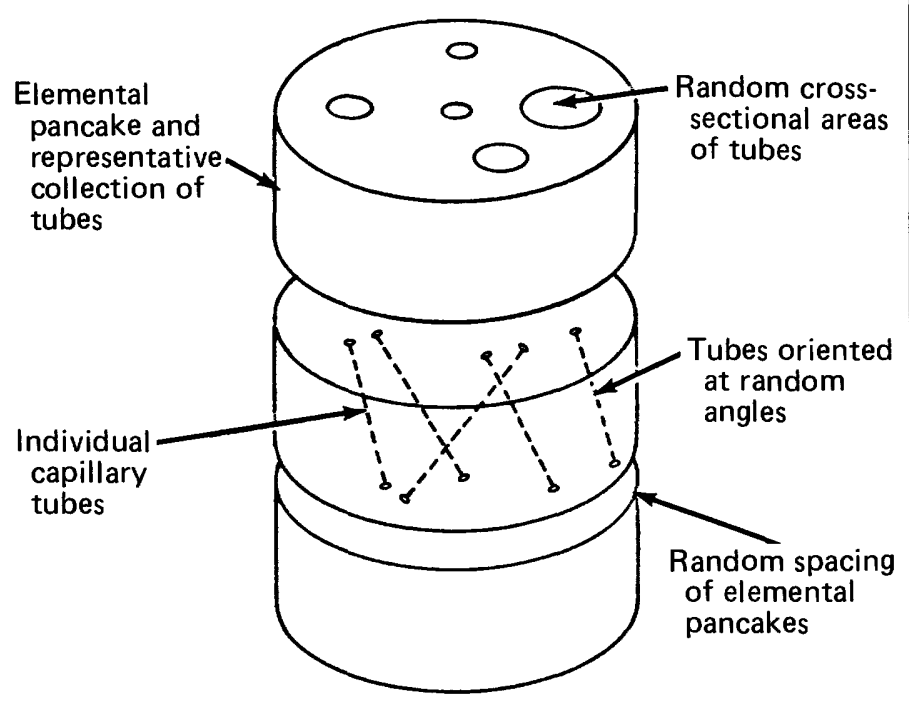

FIGURE 1.-Conceptual model of a porous body.

A second kind of pancake can be made by having the tubes of the same diameter but inclined or oriented in a random fashion, and a third arrangement can be made by having the separations between the pancakes reflect a Gaussian distribution. These concepts presuppose complete vertical continuity of flow from one pancake to another.

Thus, starting with a single tube, it is possible to build up a structure by a sequence of probability distributions that leads to the simulation of a porous body. The final description of the entire flow field, which can be calculated from the Gaussian distributions, hinges on and is determined by the behavior of the fluid moving through a single capillary tube.

In his discussion of mathematical models, Garding $(1977$, p . 6) remarks that physicists tend to validate their models by measurements in the real world; mathematicians, on the other hand, use rules of logic.

With respect to physical measurements, W. O. Smith (oral commun., 1968) noted that he was satisfied with the laboratory measurements he made on capillary tubes using an apparatus substantially as shown in figure 2. Capillary tubes and undisturbed samples of porous bodies were the subjects of extensive laboratory experiments conducted by Smith. Unfortunately, his numerical data were not organized and published prior to his death and, therefore, only this author's mathematical analysis is described in this paper.

The analytical technique adopted by this author is one that features a study of the dynamic responses of fluid in a single capillary tube subjected to the specific stimuli of selected hydraulic pressure-gradient functions. Thus, a simple flow element is selected-namely, a small slug or cylinder of water; it has already been shown that there are various ways of constructing the flow field for a porous body from such a simple element. The equations that are developed to describe the behavior of the simple flow element are then carried through for application to the whole porous body.

The foregoing procedure has a certain resemblance to differential calculus, whereby complicated volumetric structures are obtained by integration from elemental volumes. The procedure also is similar to finite-element programming, in which the properties of the element are known and are combined into larger structures by rules that define the interconnections.

\section{MATHEMATICAL ANALYSIS}

Consider the experimental flow system consisting of a horizontal capillary tube (fig. 2). The advantage of this orientation is that the elevation component of the hydraulic head is removed from the calculations. The outlet end of the tube vents to the atmosphere so that a constant pressure (zero reference) prevails at least for the duration of a given experiment. The inlet end of the tube is in a closed water-supply tank or reservoir provided with an automatic control system that allows the 
internal pressure to be changed with respect to a time function as may be prescribed for the experiment.
3. instantaneous imposition of a ramp-function change (increase or decrease) in the pressure gradient.

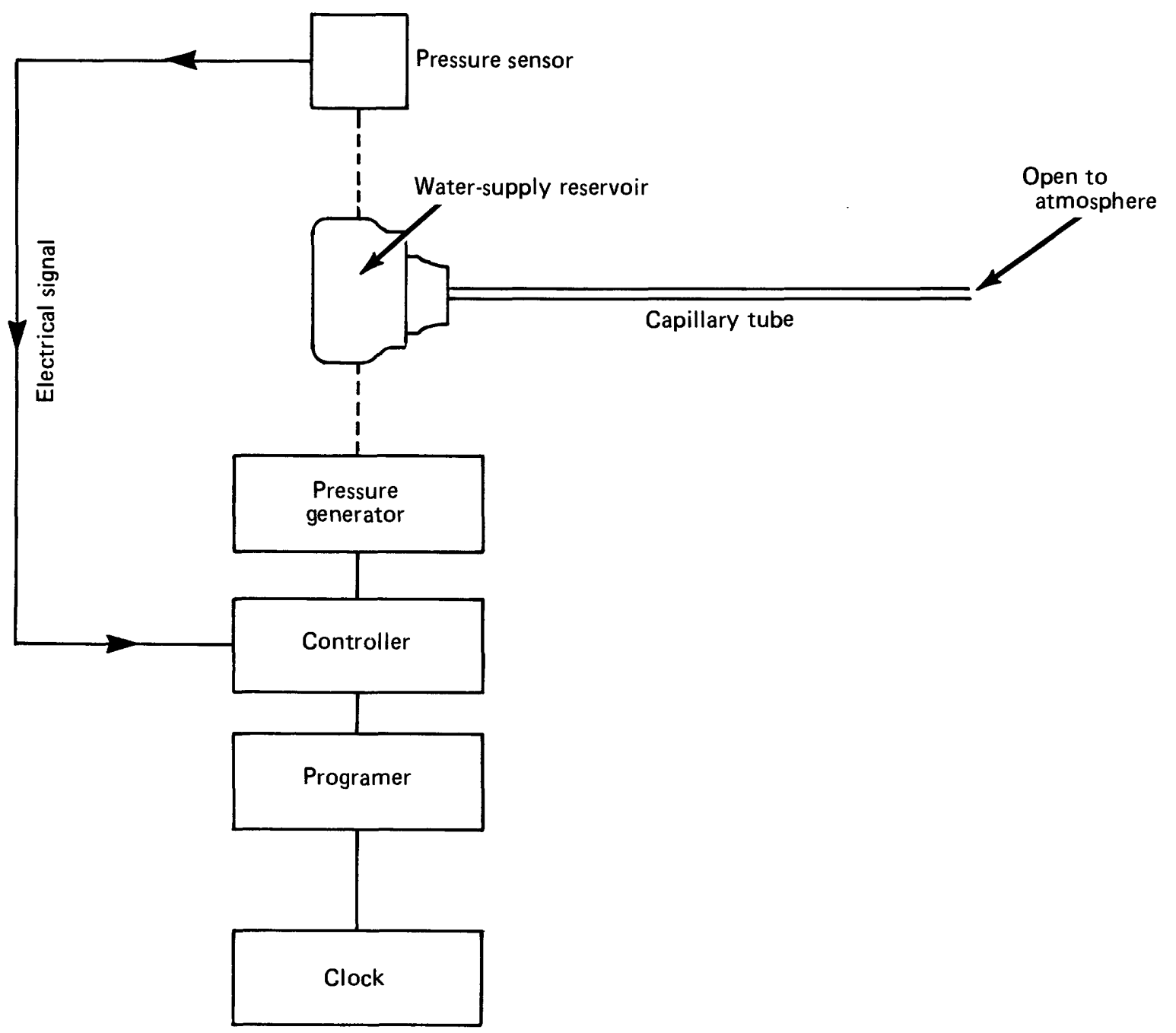

FIGURE 2.-Apparatus for producing selected hydraulic pressure-gradient functions.

The experimental setup, for which the mathematical analysis is developed, would consist of (1) initially establishing a suitable inlet pressure, (2) allowing the system to operate until steady-state equilibrium is achieved, and (3) imposing on the reservoir, at an arbitrarily chosen reference time $t=0$, any one of the following three pressure-gradient conditions or any combination thereof:

1. instantaneous release of reservoir pressure to atmospheric pressure, which allows flow system to "decay" exponentially;

2. instantaneous imposition of a step-function change (increase or decrease) in the pressure gradient; and
To describe the operation of this experimental flow system mathematically, the Navier-Stokes equations are used; they are deemed valid because they are derived directly from Newton's basic laws.

It is well known that the Navier-Stokes equations are analytically intractable. It is necessary to make some adaptation to reduce them to a form that can be solved.

According to certain observations by Reynolds (1883), in the laminar-flow state, a particle of fluid in a tube moves in a straight line. This is a physical observation that can be expressed in mathematical terms. If this is done, the Navier-Stokes equation (Aris, 1962, p. 182) is simplified and can be written as 


$$
\frac{d V_{x}}{d t}=-\frac{1}{\varrho} \frac{d p}{d x}+\nu\left[\frac{1}{r} \frac{d V_{x}}{d r}+\frac{d^{2} V_{x}}{d r^{2}}\right]
$$

where, in addition to terms previously defined,

$V x=$ fluid velocity, axial direction of tube,

$r=$ radial distance from tube axis or centerline, cylindrical coordinates,

$t$ = elapsed time,

$\nu=\underline{\mu}$, kinematic viscosity of fluid,

$x=$ distance along tube axis or centerline, and

$p=$ hydraulic pressure.

Inspection of the simplified Navier-Stokes equation shows that it is of the parabolic type (Hadamard, 1952, p. 38). The boundary conditions require that there be a statement of the initial condition and two statements regarding the fluid velocity at two different points in space. The initial condition used is simply the statement that the velocity of a fluid particle is a function of its radial distance, $r$, from the tube axis. This has the mathematical form

$$
V_{x}=-\frac{\mathrm{B}_{0}}{4}\left(a^{2}-r^{2}\right)
$$

where

$B_{0}=$ preexisting steady pressure gradient at time $t=0$ and

$a=$ radius of flow tube.

The velocity at the wall is zero at all times. This meets the requirement for one spatial statement:

$$
V_{x}(a, t)=0 .
$$

The other spatial statement comes from an examination of the conditions on the tube axis, which reveals that the velocity at $r=0$ is a singular point (see Powers, 1979, p. 21). However, for physical reasons, we know that the velocity is finite and we can therefore write

$$
V_{x}(0, t)<C,
$$

where $C$ is some constant. The desired spatial statement, however, is distilled from this by noting that on the tube axis, the velocity gradient $d V_{x} / d r$ is zero at all times, and we can write

$$
\frac{d V_{x}}{d r}(0, t)=0
$$

The problem is now well presented.

The equation is similar to the equations describing the flow of heat and also diffusion. There are several methods available for solving the simplified Navier-
Stokes equation, including the transform method or the separation of variables; a numerical solution also may be developed. A solution for the case of a step-function drive and zero initial conditions was obtained by Holland and Brenner (1965) by the application of the separation of variables technique. There seems to be an advantage in using a transform technique-in particular, a finite Hankel transformation. The conditions along the axis of symmetry of the tube and the no-slip condition at the wall are the same for many cases. For this analysis, a finite-transform technique was used; special use also was made of a Bessel function as a kernel. This technique has certain advantages when applied to this particular problem, because the initial and boundary conditions can be fitted very precisely to the Bessel functions.

The partial differential equation to be solved (see steps $1-4$ in "Appendix") is

$$
\frac{d^{2} V_{x}}{d r^{2}}+\frac{1 d V_{x}}{r d r}=\frac{d V_{x}}{d T}+\frac{1}{\mu}\left[G_{1}+G_{r} \frac{T}{v}\right]
$$

where

$T=t$, a selected change in time scale (see step 3 in "Appendix");

$G_{1}=$ a step-function pressure-gradient change; and $G_{r}=$ a ramp-function pressure-gradient rate of change. If we now operate on equation 9 and take the finite Hankel transform, as expressed by Sneddon (1951) and in table 1 , we obtain

$$
\frac{d \bar{V}}{d T}+K_{i}^{2} \bar{V}=-\frac{1}{\mu} \frac{a}{K_{i}} J_{1}\left(a K_{i}\right)\left[G_{1}+G_{r} \frac{T}{v}\right]
$$

TABLE 1.-Short table of finite Hankel transforms [Sneddon, 1951, p. 83, 531]

\begin{tabular}{ll}
\hline Real domain & Transformed domain \\
\hline${ }^{1} C$ & $\frac{a C}{K_{i}} J_{1}\left(a K_{i}\right)$ \\
$a^{2}-r^{2}$ & $\frac{4 a}{K_{i}^{3}} J_{1}\left(a K_{i}\right)$ \\
$\frac{d^{2} V_{x}}{d \mathrm{r}^{2}}+\frac{1}{r} \frac{d V_{x}}{d r}$ & $-K_{i}^{2} \bar{V}$ \\
\hline
\end{tabular}

${ }^{1} \mathrm{C}=$ constant.

where

$\bar{V}=$ fluid particle velocity in the transformed domain, $K_{i}=$ symbol denoting order of roots used in Hankel transform, and 
$J_{1}=$ Bessel function, first order, first kind,

The operation of "taking the transform" causes one variable to be "integrated out" (Sneddon, 1972, p. 10). Because $r=0$ and $r=a$ are the limits of integration, the spatial boundary conditions are automatically entered. Equation 10 reflects the results of these operations.

Equation 10 is an ordinary differential equation, to be integrated with respect to the variable, $T$, and with initial conditions available to determine the constant of integration. Recall that the initial conditions are a parabolic velocity distribution which is transformed by applying the results in table 1 to equation 5 .

Integration equation 10 with respect to $T$ gives

$$
\begin{array}{r}
\bar{V}=\frac{-1}{\mu}\left\{\left[\frac{B_{0} a}{K_{i}^{2}} J_{1}\left(a K_{i}\right)\right] e^{-b T}\right. \\
+\frac{D G_{1}}{b}\left[1-e^{-b T}\right]+\frac{D G_{r}}{b v}\left[T-\frac{1}{b}\left(1-e^{-b T)}\right]\right\},
\end{array}
$$

where

$$
\begin{aligned}
& b=K_{i}^{2} \text { and } \\
& D=\frac{1}{\mu} \frac{a}{K_{i}} J_{1}\left(a K_{i}\right) .
\end{aligned}
$$

The initial conditions have been incorporated through evaluation of the constant of integration. This equation, in the transformed domain, shows the time evolution of the velocity for all cylindrical tubes. This has the advantage, therefore, of removing the space variable and describing the transient situation with respect to the one variable of time. This equation form can reveal the time evolution of the flow-velocity profile.

The inverse finite Hankel transformation (Sneddon, 1951, p. 83) can now be performed on equation 11 to yield the following desired solution in the real domain:

$$
\begin{aligned}
V_{x}(r, t)= & -\frac{a^{2}}{\mu}\left\{B _ { 0 } \left[2 \sum_{i} \frac{J_{0}\left(\alpha_{i} z\right)}{\alpha_{i}^{3} J_{1}\left(\alpha_{i}\right)} \mathrm{e}^{\left.-t / T_{c i}\right]}\right.\right. \\
& -G_{1}\left[\frac{1-z^{2}}{4}-2 \sum_{i} \frac{J_{0}\left(\alpha_{i} z\right)}{\alpha_{i}^{3} J_{1}\left(\alpha_{i}\right)} e^{\left.-t / T_{c_{i}}\right]}\right. \\
& -G_{r}\left[\frac{1-z^{2}}{4} t-2 T_{c_{i}} \sum_{i} \frac{J_{0}\left(\alpha_{i} z\right)}{\alpha_{i}^{3} J_{1}\left(\alpha_{i}\right)}\left(1-e^{\left.-t / T_{c_{i}}\right)}\right]\right\}
\end{aligned}
$$

where, in addition to terms previously defined,

$z=r / a$, radial distance as a fraction of tube radius; $T_{c_{i}}=\frac{\varrho}{\mu} \cdot \frac{a^{2}}{\alpha_{i}^{2}}$ time constant, $i$ th term;
$J_{0}=$ Bessel function, zero order, first kind; and

$\alpha_{i}=i$ th root, Bessel function; zero order, $J_{0}\left(\alpha_{i}\right)=0$.

\section{INTERPRETATION OF TRANSIENT-FLOW EQUATION}

Equation 12 has a structure that may be interpreted from mathematical and physical points of view. The most logical approach seems to warrant looking first at some of the mathematical features. As opportunities arise, however, physical interpretations are interwoven.

The dependent variable, $V_{x}$, is by definition the fluid velocity in a direction parallel to the axis of the tube at any given point a radial distance, $r$, from that axis. $V_{x}$ is also a function of time, and thus its complete range of definition extends from $r=0$ to $r=a$ in the space domain and from $t=0$ to $t=\infty$ in the time domain.

In examining equation 12 , the velocity, $V_{x}$ is seen to comprise the sum of three bracketed terms which, in the order given, describe (1) a component related solely to the preexisting (prior to $t=0$ ) steady pressure gradient, $B_{0}$, and to the exponential decay of the flow system after that gradient is dropped to zero; (2) a component related solely to the imposition of a pressure-gradient step function, $G_{1}$; and (3) a component related solely to the imposition of a pressure-gradient ramp function, $G_{r}$. This suggests a very desirable versatility when it comes to analyzing practical problems in the field. Almost any conceivable pressure-gradient pattern should be amenable to analysis by subdividing it into the best possible arrangement of equivalent pieces that represent $B_{0}, G_{1}$, and $G_{r}$ pressure-gradient terms. Through the principle of superposition, a valid analysis can be made. Furthermore, this versatility of equation 12 is not contingent on the initial conditions being zero.

It is of interest to note that a "snapshot" of the pointvelocity profile across the tube radius during a transient condition is not a parabola. Nor is a series of such snapshots the case of a small parabola growing exponentially into a large parabola. Instead, there is throughout the evolutionary process a slight warpage away from the parabolic form. Although this deviation may be small, there is the view expressed by Birkhoff (1960) that, in hydrodynamics, there are paradoxes whereby (1) symmetrical causes do not always produce symmetrical effects and (2) small causes sometimes produce large effects. Thus, it seems appropriate to carry the fine structure of the point-velocity analysis to the useful limits afforded by equation 12 before combining the results with any further mathematical operations.

Equation 12 should first be tested to illustrate that the initial (at $t=0$ ) and boundary conditions have indeed been satisfied. If $t=0$, each exponential term equals 
unity and, therefore, a simple inspection of equation 12 shows that the third bracketed term is zero. There now remains, in each of the first and second bracketed terms, a series summation, the limiting value of which (when $t=0)$ was given by Bowman $(1958, \mathrm{p} .17)$ as $\left(1-z^{2}\right) / 4$. This shows that for $t=0$, the second bracketed term also is zero and, therefore, equation 12 reduces to the simple form

$$
V_{x}(r, t)=-B_{0} \frac{a^{2}}{\mu} \frac{1-z^{2}}{4}
$$

By inspection, equation 13 satisfies the two stated spatial boundary conditions if it is noted that the expression $d V_{x} / d r$ is zero when $z=0$ (flow-tube center) and that the velocity, $V_{x}$, is zero when $z=1$ (flow-tube wall).

An assessment of the individual significance of the three bracketed terms (and their coefficients) in equation 12 is now presented.

SIGNIFICANCE OF FIRST BRACKETED TERM

Expansion of the indicated series summation may be written as

$$
\begin{gathered}
2 \sum_{i} \frac{J_{0}\left(\alpha_{i} z\right)}{\alpha_{i}^{3} J_{1}\left(\alpha_{i}\right)} e^{-t / T_{c_{i}}}=\frac{2 J_{0}\left(\alpha_{1} z\right)}{\alpha_{i}^{3} J_{1}\left(\alpha_{1}\right)} e^{-t / T_{c_{1}}}+\frac{2 J_{0}\left(\alpha_{2} z\right)}{\alpha_{2}^{3} J_{1}\left(\alpha_{2}\right)} e^{-t / T_{c_{2}}} \\
+\frac{2 J_{0}\left(\alpha_{3} z\right)}{\alpha_{3}^{3} J_{1}\left(\alpha_{3}\right)} e^{-t / T_{c_{3}}} \ldots+\frac{2 J_{0}\left(\alpha_{n} z\right)}{\alpha_{n}^{3} J_{1}\left(\alpha_{n}\right)} e^{-t / T_{c_{n}}}
\end{gathered}
$$

As the index $i$ increases by unit steps, the successive roots $\alpha_{i}$ become larger when evaluating the series summation for a given time, $t$. (See values given on fig. 3.) In other words, successive exponential terms become smaller, and if the time factor is now allowed to increase the combined effect is that "spectral terms" of higher order decrease faster than those of lower order. Thus, the "fine structure" in the velocity profile disappears early, leaving the first term in the series summation (eq. 14) as the principal descriptor of the transient-flow condition.

An important aspect in the analysis of transient flow is the action of the inertial terms as exhibited by the time constants. The numerical value of the time constant for a simple tube representing a pore can be calculated. The value of the time constants changes enormously with the radius of the tube. The transient process-that is, the evolution of the velocity patterncan be visualized as starting at the tube wall and proceeding inward to the axis. This mode of analysis provides additional insight into the transient process.
A partial insight into the relative significance of the first three terms in the series summation of equation 14 is gained from the data plotted as figure 3 , although they were developed primarily to show how the time constant, $T_{c_{i}}$, increases with an increase in flow-tube radius. By definition, the parameter $T_{c_{i}}$ is a function of $\varrho, \mu, a$, and $\alpha_{i}$ in the manner repeated in figure 3. A dimensional analysis of $T_{c_{i}}$ shows that it does indeed have the dimensions of time, and numerical values can be computed for any fluid if $\varrho$ and $\mu$ have been determined. To prepare figure 3 , values of $\varrho$ and $\mu$ were taken for distilled water at $68^{\circ} \mathrm{F}\left(20^{\circ} \mathrm{C}\right)$ and values of the time constant, $T_{c_{i}}$, and then computed for selected tube radii-first for the Bessel function root $\alpha_{1}$ from the first term of equation 14, next for the root $\alpha_{2}$ from the second term of equation 14, and finally for the root $\alpha_{3}$ from the third term of equation 14 . The three curves plotted in figure 3 graphically illustrate the dominant importance of the first term in equation 14-an importance that is considerably strengthened by noting that the Bessel roots, $\alpha_{i}$, appear to the cubed power in the term denominators in that series-summation equation. Thus, the value of $\alpha_{l}^{3}$ is only about 14 in the first term denominator, whereas, it has grown to about 648 , or nearly fifty fold, in the third term.

The physical significance of just the first bracketed term (and its coefficient) in equation 12 can be demonstrated by neglecting, for the moment, the second and third bracketed terms by assuming that both $G_{1}$ and $G_{r}$ are equal to zero. By definition, $B_{0}$ is the preexisting steady-pressure gradient maintained on the flow system up to time $t=0$. In the experimental setup shown in figure 2 , this presumes that steady pressure (and the necessary amount of fluid) has been maintained in the water-supply reservoir, which has a very large volume relative to the tube, at some convenient value above atmospheric pressure, for a long enough period so that the rate of flow through the capillary tube has stabilized and reached an unvarying or steady state. At time $t=0$, the pressure in the reservoir is instantaneously lowered to atmospheric. Nothing more is done-no energy is externally added to or subtracted from the flow system, and the system is simply allowed to "run down." This is a classic exponential decay situation, and the exponential term in the first bracketed term of equation 12 precisely describes the manner in which the velocity, $V_{x}$, "bleeds off." As $t \rightarrow \infty$, the value of the exponential term (and hence $\left.V_{x}\right) \rightarrow 0$. Prior to $t=0$, the fluid flowed through the tube at a steady rate, and a certain level of kinetic energy obviously was being maintained. With removal of the constant pressure gradient at $t=0$, the dissipative processes set in and the kinetic energy, acting through the viscous forces, converted to heat, which decreased fluid velocity. 


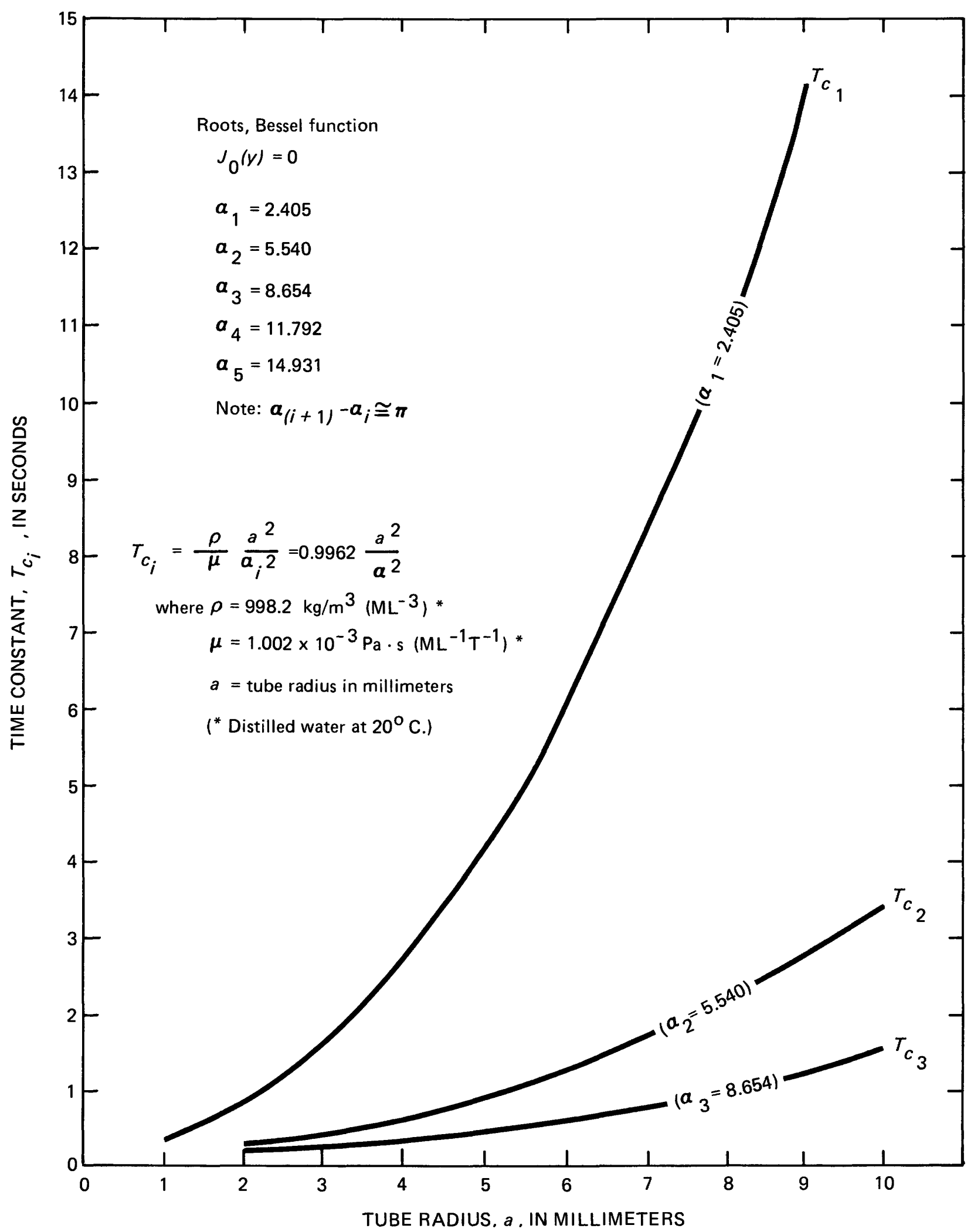

FIGURE 3.-Relation between time constant and radius of flow tube. 


\section{SIGNIFICANCE OF SECOND BRACKETED TERM}

To examine the significance of this term, neglect the first and third bracketed terms of equation 12 by assuming that $B_{0}$ and $G_{r}$ are equal to zero. Physically, this means that, prior to time $t=0$, the flow system for the experimental setup of figure 2 is quiescent at atmospheric pressure-that is, there is no pressure gradient on or flow through the capillary tube. At time $t=0$, a step-function pressure gradient, $G_{1}$, is applied to the tube by instantaneously raising the pressure in the water-supply reservoir to some convenient value above atmospheric pressure and by holding that imposed pressure constant thereafter. Holding the pressure constant means that energy (and fluid) must be fed continuously into the flow system. The supplied energy is accounted for in two ways: (1) energy that must overcome inertia and accelerate the fluid is stored as momentum, and (2) the remaining energy that must overcome the viscous forces in the fluid is dissipated as heat. If the rate of energy dissipation has increased to a value that equals the constant rate at which energy is being fed into the flow system, no further acceleration of the fluid can occur and the system has reached a steady state.

The second bracketed term of equation 12 contains the identical series summation that has already been shown, in its expanded form, as equation 14. Discussion of that equation showed that, as time elapses, the value of the summation decreases and very quickly approaches zero. As this happens, the only term left in the brackets is $\left(1-z^{2}\right) / 4$, which contains no time factor. Thus there is no further change with time-that is, the steady, or unvarying, state has been reached.

\section{SIGNIFICANCE OF THIRD BRACKETED TERM}

To examine the unique significance of this term, neglect the first and second bracketed terms of equation 12 by assuming that $B_{0}$ and $G_{1}$ are equal to zero. As in the preceding discussion, this means that, prior to time $t=0$, the flow system or the experimental setup of figure 2 is quiescent at atmospheric pressure. At time $t=0$, a ramp-function pressure gradient, $G_{r}$, is applied to the tube by raising the pressure in the water-supply reservoir at a steady rate. The nature of the flow system response can be addressed by noting that the series summation is now a bit different from that exhibited in both the first and second bracketed terms of equation 12 . Although the expansion of the series summation has the same form as that shown as equation 14 , the multiplier of each expanded term is $T_{c_{i}}\left(1-e^{-t / T} c_{i}\right)$. As time increases, this term in the series quickly approaches zero and, thus, the summation expansion reverts to the simpler form:

$$
\begin{gathered}
2 T_{c_{i}} \sum_{i} \frac{J_{0}\left(\alpha_{i} z\right)}{\alpha_{l}^{3} J_{1}\left(\alpha_{i}\right)}\left(1-e^{\left.-t / T_{c_{i}}\right)} \simeq \frac{2 T_{c_{1}} J_{0}\left(\alpha_{1} z\right)}{\alpha_{l}^{3} J_{1}\left(\alpha_{1}\right)}\right. \\
+\frac{2 T_{c_{3} J_{0}\left(\alpha_{2} z\right)}}{\alpha_{2}^{3} J_{1}\left(\alpha_{2}\right)}+\frac{2 T_{c_{3} J_{0}\left(\alpha_{3} z\right)}^{\alpha_{3}^{3} J_{1}\left(\alpha_{3}\right)}}{+} \\
+\frac{2 T_{e_{n} J_{0}\left(\alpha_{n} z\right)}^{\alpha_{n}^{3} J_{1}\left(\alpha_{n}\right)}}{}
\end{gathered}
$$

The root characteristics of the Bessel function $J_{0}$ and $J_{1}$ resemble a cosine function and a sine function, respectively. Their combined effect, as successive terms in the series summation (equation 15) are evaluated, is to approach a finite limit. This occurs after only the first two or three terms are used; however, each still needs to be multiplied by the appropriate time-constant parameter, $T_{c_{i}}$. Furthermore, a time parameter, $t$, also is the multiplier for the first term appearing in the third bracketed term of equation 12 .

One way of visualizing what is described by the third bracketed term of equation 12 as time elapses is to say that the response, $V_{x}$, although trying to catch up with the stimulus, $G_{r}$, succeeds only in approaching a finite lag for any given radial distance, $r$, from the flow-tube axis. This finite lag is different for each radial distance. Thus, the velocity profile "warps" with the time. Because the flow system has inertia, the response can never completely catch up with the stimulus, which is increasing continuously at a steady or linear rate.

Interpretation of the transient-flow equation is not complete without elaborating on the nature of the time constant, $T_{c_{i}}$. This is a key parameter in characterizing the transient-flow process.

The time constant, as calculated from its definition in equation 12 , reflects the nature of the assumptions made in devising a single capillary-tube model. Thus, it would not be expected to yield numerical values that necessarily agreed with similar constants determined from other investigators' works, inasmuch as the latter are based on different sets of modeling assumptions.

The time constant may be regarded as the time interval that extends from the moment a linear time invariant system is perturbed by, say, a step function until the system has reached 63 percent of the new steadystate condition. A time invariant system is one whose properties, such as dimensions, viscosity, density, and temperature, are constants. A linear system is one to which the mathematical principle of superposition applies. Time constants appear in the exponential terms in the transient-flow equation; their significance may 
be illustrated by three distinct fluid-flow situations:

1. time constant is very short compared with the duration of the flow process,

2. time constant is of the same order as the duration of the process, and

3. time constant is long compared with the process duration.

In the first case, which is common in geology, the transient flow effects can be neglected; in the second case, those flow effects should be estimated in order to make a good approximation; and in the third case, transient flow effects must be taken into account to provide a realistic view of the whole flow process.

\section{COMPARISON WITH DARCY'S FLOW EQUATION}

A method of comparing the analytical results obtained from the Darcy (steady-state) and transient-flow equations is computing the volume rate of discharge for a given flow condition with each of the two equations (fig. 4). Consider a representative sample of a porous body
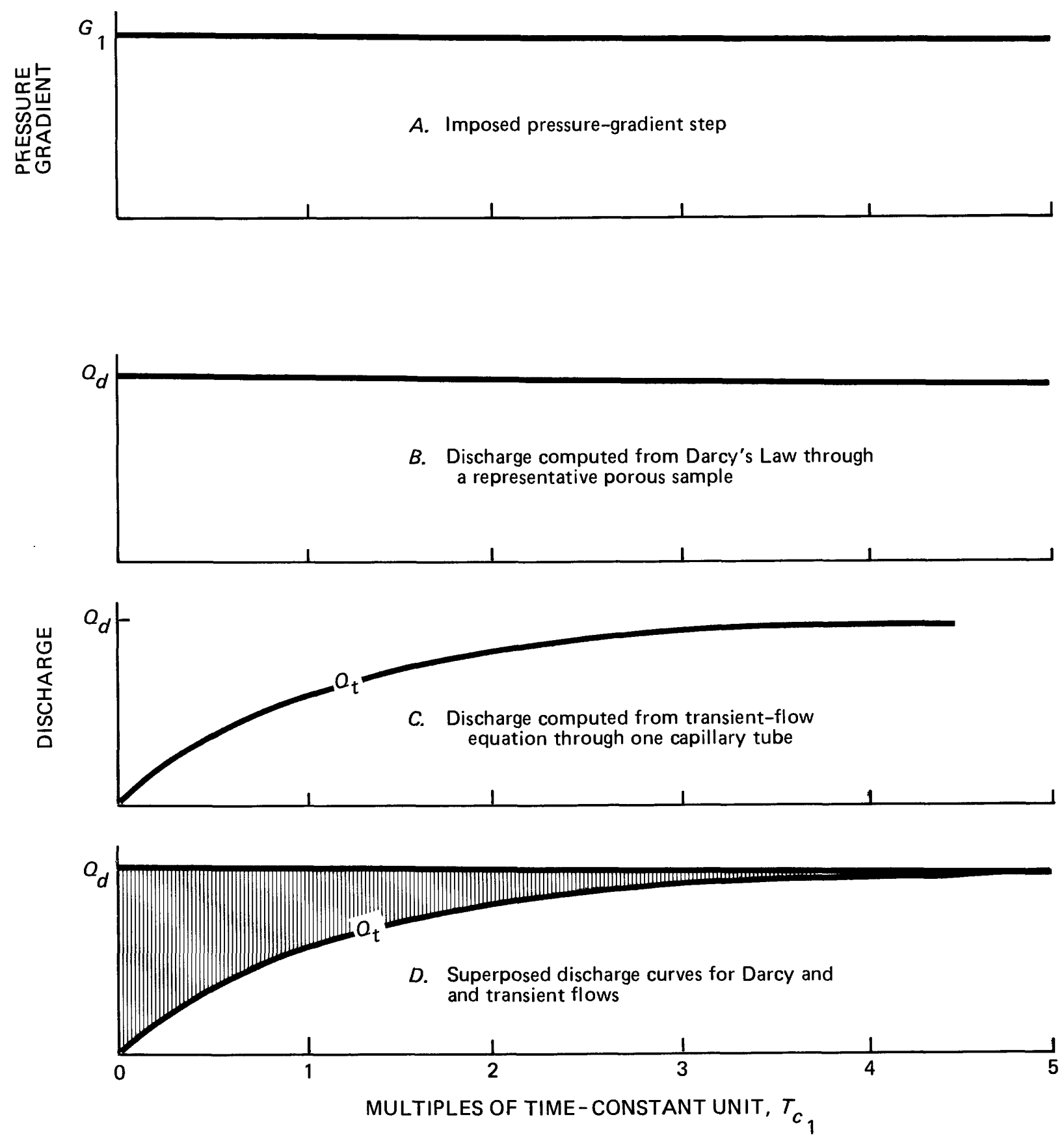

FIGURE 4.-Comparison between Darcy and transient-flow equations for indicated step increase in pressure gradient. 
initially quiescent and then subjected to a step-function pressure gradient, $G_{1}$, (fig. $4 A$ ). From Darcy's law, an expression for the volume rate of discharge, $Q_{d}$, can be written directly from equation 1 in the form

$$
Q_{d}=-K A \frac{G_{1}}{\varrho g},
$$

where

$$
\frac{G_{1}}{\varrho g}=\frac{h_{2}-h_{1}}{L} \text {. }
$$

Equation 16 contains no time factor and obviously describes an unvarying or steady-state discharge condition (fig. 4B).

Now consider a capillary tube of radius $a$, in a quiescent state, and then apply the same step-function pressure gradient $\left(G_{1}\right)$. No other change is imposed, and the response is uniquely described by simply the second bracketed term (and its coefficient) of equation 12, which defines, as a function of time, a velocity profile at right angles to the tube axis. This term can be integrated as a function of radial distance from the tube centerline, between the limits $r=0$ and $r=a$. This yields the following expression for the volume rate of discharge, $Q_{t}$, as a function of time:

$$
Q_{t}=\frac{\pi a^{4} G_{1}}{8 \mu}\left[1-\frac{32}{\alpha_{1}^{4}} e^{-t / T_{c_{1}}}\right]
$$

Because the series summation in the referenced term of equation 12 converges rapidly, only the first term of the expansion needed to be integrated. Also, because of $\alpha_{1}{ }^{4}$ equals 33.2 , the coefficient of the exponential term in equation 17 can be replaced with the value 1 . Equation 17 may, therefore, be rewritten in the form

$$
Q_{t}=\frac{\pi a^{4} G_{1}}{8 \mu}\left[1-e^{-t / T_{c_{1}}}\right]
$$

Equation 18 may, therefore, be regarded as a mathematical model for the transient-flow state showing the discharge response from a capillary tube subjected to a step-function pressure gradient.

If the value of $t$ becomes sufficiently large compared with $T_{c_{1}}$, the exponential term in the brackets (equation 18) becomes negligible. Indeed, if $t=5 T_{c_{1}}$, the value of the exponential term is less than 0.02 . Thus, for any larger values of $t$, the bracketed part of equation 18 may be considered equal to unity, and, thus, $Q t$ has reached an unvarying or steady state. The foregoing observations are readily apparent in figure $4 C$, which is a plot of equation 18.

To compare the difference between the two preceding methods for computing rate of discharge under the stipulated flow conditions, the discharge curve shown in figure $4 C$ is superposed on the curve shown in figure $4 B$. Note, however, that the curve in figure $4 C$ represents the rate of discharge through just one capillary tube. Because this single tube is representative of all tubes, it is permissible to multiply the rate of discharge in the one by some number, $n$ (number of tubes), such that the combined or total rate of discharge exactly equals the steady rate of discharge (graphed in fig. $4 B$ ) for the representative sample of the porous body when steady state is reached.

The superposed results are as shown in figure $4 D$; the hachured area indicates the difference between the two forms of analysts during the transient or unsteady part of the flow period. Observe that the two curves are indeed coincident when the elapsed time, $t$, has increased to $5 T_{c_{1}}$. Thereafter, the two forms of analysis yield identical results because steady-state conditions prevail in the flow system.

For an aquifer of medium gravel, in which the average pore-space radius might be $1 \mathrm{~mm}$, the corresponding value of $T_{c_{1}}$, as read from figure 3 , is about 0.5 second. This suggests that, in response to some step-function change imposed on the aquifer, the duration of the transient flow period would be about 2.5 seconds.

The validity of the preceding discussion, which compares the results obtained from the Darcy equation rather than the transient-flow equation, is restricted in no way by the arbitrary choice of a step-function pressure gradient as the stimulus to the flow system. The choice was primarily for purposes of illustration.

\section{SUMMARY AND CONCLUSIONS}

The mathematical analysis in this paper is based on equation 4 . If we multiply each term in that equation by the fluid density, $\varrho$, the left-hand side represents a change in the momentum or a force. The first term on the right-hand side represents the causative force provided by the pressure gradient. The last term on the right-hand side can then be regarded as the dissipative or opposing force due to viscosity-in effect, a frictional force. Hence, this version of equation 4 is a "balance of forces" equation.

Consider each term of the foregoing version multiplied by a velocity, $V_{x}$. The left-hand side of the resulting equation may be interpreted to mean the change in kinetic energy. The first term on the right-hand side represents energy input per unit time to maintain the pressure gradient. The second term on the right-hand side gives the rate at which frictional energy is dissipated as heat. We now have a representation of the fluid-flow process in terms of energy. In the steady state, the kinetic-energy term is constant-that is, energy is being introduced into the system at a rate just sufficient 
to compensate for the frictional losses. The advantage in considering the fluid-flow process from this point of view-a balance in the rates of energy flow-is that it gives a further insight into the transient as well as the steady-state flow conditions.

In a fluid-flow system, the essence of a transient process is its time evolution. Therefore, the time variable is normally the dominant parameter in any mathematical description of that system. In this report, the measure of time that characterizes the continuously changing fluid-flow process is identified as the time constant, and it first appears in equation 12 . If the description of the transient process is given in terms of the time constant, the time evolution is independent of real time. Therefore, it is a simple matter to use equation 12 to analyze specific flow-field problems.

A closer look at the structure of the time constant is appropriate. The time constant obviously includes two kinds of information; the first relates to the properties of the fluid ( $\mu$ and $\varrho$ ), and the second relates to the geometry of the flow tube (radius $r$, and Bessel root, $\alpha_{i}$, which implies cylindrical shape). The dynamic viscosity, $\mu$, of the fluid is associated with the dissipative action in the flow system, and the fluid density, $\varrho$, is associated with the storage of kinetic energy. Thus, for any given flow system, the time constant must reflect the ratio between the storage of kinetic energy and the dissipative action, inasmuch as it includes the ratio of $\varrho$ to $\mu$.

On the basis of mathematical analysis in this paper, equation 12 is shown to be a convenient tool for examining specific ground-water situations, regardless of whether they are simulated in the laboratory or encountered in the field. Equation 12 includes pressuregradient terms that are measures of energy input. In the laboratory, such inputs can be precisely controlled as to amplitude and time of application. Then, the response is predictable and reflects the design of the apparatus. Similar controlled inputs can be made in the field environment. Thus, an aquifer-recharge experiment of a selected magnitude can be started at any given moment and with any convenient well. The response will be predictable and will reflect the known physical properties and dimensions of the porous-earth material comprising the aquifier. Depending on the nature of the experiment-laboratory or field-the numerical value of the time constant can be determined (core sample analysis) and a judgment made as to the merits of using the transient-flow analysis.

The time constant increases as the square of the radius of the capillary flow-tube, which in turn is intended to represent a flow path through some porous earth material. If a tube radius of $3 \mathrm{~mm}$, for example, is selected (that radius would be exceeded in the field only by the pore radius of very coarse gravels or cavernous limestone), the time constant would be about 1.7 seconds (fig. 3). Thus, if a step-function type of pressure gradient were applied to this size of flow tube, the steady-state condition would be reached in about 8.5 seconds. This helps to place a practical upper limit on the value of the time constant. In other words, if a detailed description of the rate of discharge is needed within the first 8 seconds of the foregoing flow conditions, equation 12 should be used.

If a linear type of pressure-gradient change had been postulated as the basis for analysis instead of a step function, similar arguments could have been made with only some differences in detail, the principal difference being the development of a standing time lag in the response when compared with results obtained by applying Darcy's law. The time lag would be of the order of two time constants; again, equation 12 would permit the calculation for any specific set of conditions.

It may be concluded that, for most of the commonly encountered ground-water flow systems, the simple Darcy equation remains an adequate mathematical description. The interesting point is that any reasonable set of flow-field circumstances can now be evaluated rapidly and the most appropriate analytical procedure followed. Of course, experimental verification would be highly desirable.

One final observation seems pertinent to the direction that might be taken for any further research. In the mathematical analysis given herein, the velocity profile was described by Fourier series. This is a powerful analytical technique, and its application to further studies of the "structure" of fluid flows would enhance our knowledge and understanding of the intricacies of unsteady fluid flow processes.

The analysis presented in this report suggests directions for future research. One direction would be to examine the behavior of fluid flow through assemblages of tubes and connections organized from simple elements by probability rules. Another direction would be to measure, in the laboratory, the transient response of fluid flow through a core sample of porous earth material.

\section{SELECTED REFERENCES}

Aris, Rutherford, 1962, Vectors, tensors and the basic equations of fluid mechanics: Englewood Cliffs, N.J., Prentice-Hall, 286 p.

Birkhoff, Garrett, 1960, Hydrodynamics: A study in logic, fact and similitude: Princeton, N.J., Princeton University Press, 184 p.

Blake, F. C., 1922, The resistance of packing fluid flow: American Institute of Chemical Engineers Transactions, v. 14, 415 p.

Bowman, Frank, 1958, Introduction to Bessel functions: New York, Dover Publications, 135 p. 
Carman, P. C., 1956, Flow of gases through porous media: New York: Academic Press.

Darcy, Henri, 1856, Les fontaines publiques de la ville de Dijon: Paris, Victor Dalmont.

Garding, Lars, 1977, Encounter with mathematics: New York, Springer, $270 \mathrm{p}$.

Gray, W. G., and O'Neill, Kevin, 1976, On the general equations for the flow in porous media and their reduction to Darcy's law: Water Rosources Rosearch, v. 12, no. 2, p. 148-154.

Hadamard, Jaques, 1952, Lectures on Cauchy's problem in linear partial differential equations: New York, Dover Publications, $316 \mathrm{p}$.

Happel, John, and Brenner, Howard, 1965, Low Reynolds number hydrodynamics with special applications to particulate media: Englewood Cliffs, N.J., Prentice-Hall, 553 p.

Holland John, and Brenner, Howard, 1965, Low Roynolds number hydrodynamics with special applications to particulate media: Englewood Cliffs, N.J., Prentice-Hall, 553 p.

Hubbert, M. K., 1969, The theory of ground-water motion and related papers: New York, Hafner Publishing Co., 310 p.

Kozeny, J., 1953, Hydraulik: Vienna, Springer, 390 p.
Lohman, S. W., 1972, Ground-water hydraulics: U.S. Geological Survey Professional Paper 708, 70 p.

Powers, David, 1979, Boundary value problems (2d ed.): New York, Academic Press, $351 \mathrm{p}$.

Reynolds, Osborne, 1883, An experimental investigation of the circumstances which determine whether the motion of water shall be direct or sinuous and of the law of resistance in parallel channels: London, Royal Society Philosophical Transactions, v. 174, $935 \mathrm{p}$.

Scheidegger, A. E., 1964, Statistical hydrodynamics in porous media, in Chow, V. T., Advances in hydroscience: New York, Academic Press, v. 1, p. 161-181.

Slichter, C. S., 1899, Theoretical investigation of the motion of ground waters: U.S. Geological Survey 19th annual report, pt. 2, p. 301-384.

Sneddon, I. N., 1951, Fourier transforms: New York, McGraw-Hill, 542 p.

1972, The use of integral transforms: New York, McGrawHill, 539 p. 


\section{APPENDIX: SUPPLEMENTAL DERIVATION OF EQUATIONS}

The following numbered steps show the manner in which the transient-flow equation was derived, starting from the modified Navier-Stokes equation.

1. Navier-Stokes equation simplified:

$$
\frac{\partial V_{x}}{\partial t}=-\frac{1}{\varrho} \frac{\partial p}{\partial x}+v\left[\frac{\partial^{2} V_{x}}{\partial r^{2}}+\frac{1}{r} \frac{\partial V_{x}}{\partial r}\right]
$$

2. Introduction of the time-dependent gradient, $G_{1} G_{r} t$, a simple polynomial forcing function that reflects a physically attainable state:

$$
\begin{aligned}
& \frac{\partial p}{\partial x}=G_{1}+G_{r} t \\
& \frac{\partial V_{x}}{\partial t}=-\frac{1}{\varrho}\left[G_{1}+G_{r} t\right]+\nu\left[\frac{\partial^{2} V_{x}}{\partial r^{2}}+\frac{1}{r} \frac{\partial V_{x}}{\partial r}\right] \\
& \frac{\partial V_{x}}{\nu \partial t}=-\frac{1}{\varrho \nu}\left[G_{1}+G_{r} t\right]+\left[\frac{\partial^{2} V_{x}}{\partial r^{2}}+\frac{1}{r} \frac{\partial V_{x}}{\partial r}\right]
\end{aligned}
$$

3. Insert change of time scale:

$$
T=\nu t \text { and } d T=\nu d t .
$$

4. Equation to be solved:

$$
\frac{\partial^{2} V_{x}}{\partial r^{2}}+\frac{1}{r} \frac{\partial V_{x}}{\partial r}=+\frac{\partial V_{x}}{\partial T}+\frac{1}{\mu}\left[G_{1}+\frac{G_{r}}{v} T\right]
$$

5. Boundary conditions:

a. no slip at the flow-tube wall:

$$
V_{x}[a, t]=0
$$

b. finite velocity on the flow-tube axis:

$$
V_{x}[0, t]<C
$$

c. symmetry:

$$
\frac{\partial V_{x}}{\partial r}[0, t]=0
$$

d. initial conditions:

$$
V_{x}=\frac{B_{0}}{4 \mu}\left[a^{2}-r^{2}\right]
$$

6. Equation transformed by finite Hankel transform:

$$
\frac{d \bar{V}}{d T}+K_{i}^{2} \bar{V}=-\frac{1}{\mu} \frac{a}{K_{i}} J_{0}\left[a K_{i}\right]\left[\mathrm{G}_{1}+\frac{G_{r}}{v} T\right]
$$

7. Integration of the transformed equation:

$$
\begin{aligned}
\frac{d \bar{V}}{d T} e^{K_{i}^{2} T}+K_{i}^{2} \bar{V} & e^{K_{i}^{2} T}= \\
& -\frac{1}{\mu}\left[G_{1}+\frac{G_{r}}{\nu} T\right] \frac{a}{K_{i}} J_{0}\left(a K_{i}\right) e^{K_{i}^{2} T}
\end{aligned}
$$

The primitive is:

$$
\begin{gathered}
\bar{V} e^{K_{i}^{2} T}=\int-\frac{1}{\mu} G_{1} C_{1} e^{K_{i}^{2} T} d T- \\
\int \frac{G_{r}}{\mu \nu} C_{1} T e^{K_{i}^{2} T} d T+C_{0} \\
\bar{V} e^{K_{i}^{2} T}=-\frac{1}{\mu} \frac{1}{K_{i}^{2}} G_{1} C_{1} e^{K_{i}^{2} T}- \\
\frac{G_{r}}{\mu \nu} C_{1}\left(\frac{T}{K_{l}^{2}}-\frac{1}{K_{l}^{4}}\right) e^{K_{i}^{2} T}+C_{0}
\end{gathered}
$$

8. Evaluate the constant of integration, using the transformed initial conditions.

$$
\begin{aligned}
& \text { at } T=0, \bar{V}=-\frac{B_{0}}{\mu} \frac{a}{K_{i}^{3}} J_{1}\left[a K_{i}\right] \\
& C_{0}=\frac{B_{0}}{\mu} \frac{a}{K_{i}^{2}} J_{1}\left(a K_{i}\right)+\frac{1}{\mu} \frac{1}{K_{i}^{2}} G_{1} C_{1}-\frac{1}{\mu} \frac{1}{K_{l}^{4}} G_{r} C_{1}
\end{aligned}
$$

9. Solution in the transformed domain:

$$
\begin{aligned}
V\left(K_{i} T\right)^{5} \frac{-1}{\mu}\left\{\left[\frac{B_{0} a}{K_{i}^{3}} J_{1}\left(a K_{i}\right)\right] e^{-b T}+\frac{D C_{1}}{b}\left[1-e^{-b T}\right]\right. & +\frac{D G_{r}}{b v}\left[T-\frac{1}{b}\left(1-e^{-b T)}\right]\right\} \\
b=K_{i}^{2} \quad D & =\frac{1}{\mu} \frac{a}{K_{i}} J_{1}\left(a K_{i}\right)
\end{aligned}
$$

10. Perform the inverse transformation. Use table of transform pairs and also definition of inverse transformation.

$$
\begin{gathered}
V_{x}(r t)=-\frac{a^{2}}{\mu}\left\{B _ { 0 } \left[2 \sum_{i} \frac{J_{0}\left(\alpha_{i} z\right)}{\alpha_{i}^{3} J_{1}\left(\alpha_{i}\right)} e^{-t / T_{c_{i}}}\right.\right. \\
+G_{1}\left[\frac{1-z^{2}}{4}-2 \sum_{i} \frac{J_{0}\left(\alpha_{i} z\right)}{\alpha_{i}^{\beta_{0} J_{0}\left(\alpha_{r}\right)} e^{-t / T_{c_{i}}}}\right. \\
\left.+G_{r}\left[\frac{1-z^{2}}{4} t-2 T_{c_{i}} \sum_{i} \frac{J_{0}\left(\alpha_{i} z\right)}{\alpha_{i}^{3} J_{1}\left(\alpha_{i}\right)}\left(1-e^{\left.-t / T_{c_{i}}\right)}\right)\right]\right\} \\
T_{c_{i}} \text { ith term } T_{c_{i}}=\frac{\rho \alpha^{2}}{\mu \alpha_{i}^{2}}
\end{gathered}
$$




\section{LIST OF SYMBOLS AND ABBREVIATIONS}

Symbol

A

$B_{0}$

$C_{\mathrm{o}}$

$C_{1}$

$D$

$G_{1}$

$G_{r}$

$J_{0}$

$J_{1}$

$K$

$K_{i}$

$L$

$Q$

$Q_{d}$

$Q_{t}$

$T$

$T_{c_{i}}$

$v_{x}$

$\bar{V}$

$a$

$b$

c

g

h
Dimensions

$L^{2}$

$M L^{-2} T^{-2}$

$T^{2-1}$

$L$

$M L^{-1} T^{2}$

$M L^{-2} T^{-2}$

$M L^{-2} T^{-3}$

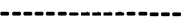

$L T^{21}$

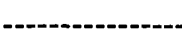

$L$

$L^{3} T^{-1}$

$L^{3} T^{-1}$

$L^{3} T^{-1}$

$L^{2}$

$L^{2}$

$L T^{-1}$

$T^{-1}$

$L$

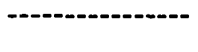

$L T^{-2}$

$L$

$L^{2}$

$M L^{-1} T^{-2}$

$L T^{-1}$
Description

Cross-sectional area of porous medium perpendicular to direction of flow.

Preexisting steady pressure gradient at time $t=0$.

Constant of integration.

Symbol for $\frac{a J_{1}\left(a K_{i}\right)}{K_{i}}$.

Symbol for $\frac{a J_{1}\left(a K_{i}\right)}{\mu K_{i}}$.

A step-function pressure-gradient change.

A ramp-function pressure-gradient rate of change.

Bessel function, zero order, first kind.

Bessel function, first order, first kind.

Hydraulic conductivity of porous medium.

Symbol denoting roots used in the Hankel transform; $J_{0}\left(a K_{i}\right)=0$.

Length of flow path.

Volumetric rate of flow.

Volumetric rate of flow calculated according to Darcy's law.

Volumetric rate of flow calculated according to equation 12.

Changed time scale; $T=v t$.

Time constant, ith term; $T=\frac{\varrho a^{2}}{\mu a_{i}^{2}}$.

Fluid velocity in axial direction of tube.

Fluid particle velocity in transformed domain.

Radius of flow tube.

Symbol for $K_{i}^{2}$

Constant.

Acceleration due to gravity.

Hydraulic head.

Index in integers.

Intrinsic permeability of porous medium.

Hydraulic pressure at a given point.

Specific rate of fluid flow. 


\section{VALIDITY OF DARCY'S LAW UNDER TRANSIENT CONDITIONS}

Dimensions

$L$

$T$

$L$

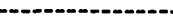

$M L^{-1} T^{-1}$

$L^{2} T^{-1}$

$M L^{-3}$

$T$

$L^{2} T^{2}$

\section{Description}

Radial distance from tube axis.

Elapsed time.

Distance along tube axis or centerline.

Radial distance as a fraction of tube radius; $z=r / a$.

Dynamic viscosity of fluid.

Kinematic viscosity of fluid.

Density of fluid.

Specific conductivity of fluid.

Fluid potential; $\phi=g h$. 